\title{
COVID-19 Virulence: Hinderances to Observing the Preventive Measures
}

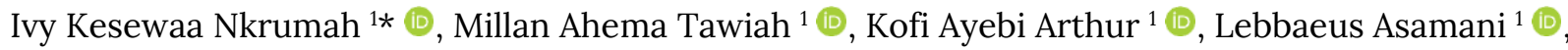 \\ Ebenezer Takyi-Wadieh ${ }^{1}$ (D)
}

${ }^{1}$ University of Cape Coast, GHANA

*Corresponding Author: ivy.nkrunmah@ucc.edu.gh

Citation: Nkrumah, I. K., Tawiah, M. A., Arthur, K. A., Asamani, L., \& Takyi-Wadieh, E. (2021). COVID-19 Virulence: Hinderances to Observing the Preventive Measures. Mediterranean Journal of Social \& Behavioral Research, 5(1), 59-66. https://doi.org/10.30935/mjosbr/11293

\begin{abstract}
The Coronavirus disease (COVID-19) is the defining global health crisis in contemporary times. To date, there is no particular cure for COVID-19 and prevention remains the chief approach in tackling the pandemic. Social distancing, hand sanitising, wearing of nose masks and handwashing have been imposed by the government of Ghana to limit the disease spread. However, research (e.g., Bonful et al., 2020) shows that some sections of Ghanaians do not fully adhere to the COVID-19 preventive measures. This study sought to examine the extent to which university students observe each of the aforementioned government-imposed COVID-19 preventive measures and the factors that impair students' efforts to observe these measures. Using a multi-stage sampling procedure, a total of one hundred and eighty-three students (88 males and 95 females) with mean age of 26 participated in the study. A cross-sectional survey design was employed for the study, and a questionnaire was used for data collection. Whereas most respondents reported wearing their nose masks and sanitising their hands quite frequently, a significant few also declined practicing these. Maintaining social distancing was the least and most infrequent practiced preventive measure. The barriers to observing the preventive measures included the number of students the university maintains in a room, unavailability of the needed materials and inappropriate positioning of the handwashing and hand sanitising stations, the expressed discomfort associated with prolonged use of facemasks, and the social behaviours of students not taking such actions in response to normative group influence. It was recommended that the university management invest more resources to increase and equip the handwashing and hand sanitising stations, modify the existing policy on room intake in the university halls and review the policy on instructional periods to facilitate student's efforts in observing the preventive measures. In addition, awareness creation in terms of billboards and screen teasers are needed on the campuses to sensitise and engender students to do the needful.
\end{abstract}

Keywords: COVID-19, barriers, preventive measures

Received: 1 Aug. 2021 Accepted: 7 Sep. 2021

\section{INTRODUCTION}

The World Health Organisation (WHO) describes COVID-19 as a contagious disease caused by a new coronavirus referred to as SARSCoV-2 (2019-CoV). COVID-19 outbreak is believed to have started in Wuhan, China in December 2019 (WHO, 2020a; Holmes, 2020). Persons infected with the COVID-19 virus show discernible symptoms such as difficulty in breathing, fever, tiredness, nasal congestion and dry cough, and these signs appear at approximately two to fourteen days after a person has been infected. The COVID-19 virus can spread from an infected person's mouth or nose in small liquid particles when they sneeze, sing, speak, cough or breathe heavily. These liquid particles appear in varied proportions, ranging from larger 'respiratory droplets' to smaller 'aerosols'. Current evidence suggests respiratory droplets among people in close contact with each other as the main way that the virus spreads. The virus can also spread after infected persons sneeze, cough on, or touch surfaces, of such objects as tables, doorknobs and handrails. Other people could become infected by touching such polluted surfaces, and later touching their eyes, noses or mouths without having cleaned their hands first. Aerosol transmission can occur in specific settings, predominantly in indoor, crowded and poorly ventilated spaces, where infected person(s) stay for long periods of time, including classrooms, restaurants, choir practices, fitness classes, nightclubs, offices and/or places of worship.

COVID-19 is hurriedly spreading across the world (Chen, Zhou, \& Dong, 2020; Li, Guan, \& Wu, 2020; Gorbalenya et al., 2020) and it has been acknowledged as a pandemic by WHO (2020a). Since there is no cure for COVID-19, appropriate alternatives are required for preventing the transmission of the virus in the form of public health and social initiatives. Currently, measures such as case isolation, contact tracing and quarantining, social distancing, foreign travel measures, and vaccinations (WHO, 2020b) have been employed by governments 
across the world. It is suggested that the most effective preventive measures have been maintaining physical distance (a minimum of 1 metre) from other people, and performing hand hygiene often with an alcohol-based hand rub, and if hands are not perceptibly dirty then wash with soap and water. Other measures include wearing a medical mask if one has respiratory symptoms and performing hand hygiene after disposing off the mask; and routine cleaning and disinfection of environmental and other frequently touched surfaces. All these preventive measures are meant to limit the spread of the virus.

As at $24^{\text {th }}$ July, 2020, Ghana's COVID-19 cases stood at 30,000 and 153 deaths, and that placed Ghana third highest, on the list of countries in Africa that had reported COVID-19 cases and 54th globally (WHO, 2020a). In spite of efforts by the government of Ghana to reduce the spread of the virus, the number of reported cases appear to be rising. The current COVID-19 statistics in Ghana shows 79766 cases and 572 deaths (Ghana Health Service, 2020), indicating that the situation of the pandemic is not improving. Due to numerous and varied sensitisation and awareness programs in Ghana concerning COVID-19, it is expected that the entire citizenry would make efforts on their part, to arrest the spread of the disease. However, it has been reported that some sections of Ghanaians especially people who find themselves in unrestricted and crowded places like the markets and lorry parks, do not fully observe the recommended safety and prevention protocols (Joy News, 2021). Even university students who are assumed to possess a better knowledge of the dynamics of the virus spread (since they have access to internet and can read more about COVID-19 happenings in other countries), have been reported as not fully adhering to the protocols (Atlantic FM, 2021). The few students who do, are selective in terms of the restrictions that are convenient to them. This perhaps, explains why government's efforts to reduce the spread of the virus has not yielded the expected outcome. It is worth mentioning that the first shot of COVID-19 vaccines arrived in Ghana on May 7, 2020, and the doses received was 350,000 (Ghana Health Service, 2021) for a population of over $31,000,000$. As at the time of this study, most people had not been served the vaccine yet and prevention remains the principal focus of COVID-19 sensitisation programmes in Ghana.

In this study, the researchers explored the extent to which university students adhere to the recommended COVID-19 protocols and more importantly, the factors that impair students' efforts in observing the protocols. The parameters of the recommended protocols of interest were washing of hands, use of hand sanitisers, wearing of nose masks and keeping social distancing. The University of Cape Coast was chosen as the study's locale because all the researchers are staff of the university and they had made such cursory observations of student's attitudes towards the protocols, as imposed by government, and enforced by the university's proctor. The main objectives of the study were to: (i) explore the extent to which students adhere to the COVID-19 protocols (handwashing, hand-sanitising, wearing of face masks and social distancing), (ii) examine the factors that impair students abilities to adhere to the COVID-19 protocol of hand washing (iii) assess the factors that impair students abilities to observe the COVID-19 protocol of hand sanitising, (iv) examine the reasons students assign to their inability to observe social distancing, (v) examine the reasons that impair students efforts in wearing nose masks in the midst of COVID-19.

\section{METHODOLOGY}

\section{Design}

The cross-sectional survey design was employed in this study because the researchers aimed at casting light on the present phenomenon of COVID-19 prevention scheme through a process of data collection that helps the researchers to describe the situation comprehensively than will be possible without using this method (Fox, 2007). The population for the study was students from the University of Cape Coast in Ghana. A total of 190 students in the university participated in the study. The participants consisted of 88 males and 95 females. The respondents belonged to the various colleges and departments in the university. Their age range was 18 to 41 with mean a mean age of 26 .

\section{Instrument}

A self-structured questionnaire, consisting of closed-ended items was the main instrument for the study. The questionnaire consisted of two sections (A and B). Section "A" sought for the demographic data of respondents in addition to two questions on duration and frequency at which respondents observed each of the COVID-19 protocols on a daily basis. Section B sought for information on the factors that impaired respondent's efforts in responding to the COVID-19 preventive measures.

\section{Procedure}

The researchers initially introduced themselves to the participants. The participants were told that the study was meant to gather information about their attitude towards the government-imposed measures for mitigating the spread of COVID-19, as it was meant to strengthen management decisions. They were assured of anonymity of their responses and this was further emphasised in print on the questionnaire. The questionnaires were distributed by the researchers among the participants and they were given one day to complete questionnaires and return them to the researchers at an agreed place. The respondents were verbally assured of confidentiality of their responses and this was emphasised in writing. All the participants were above 18 years so they provided informed consent by themselves.

\section{Population}

The University of Cape Coast was chosen as the study's locale because one of the reports on non-adherence to COVID-19 protocols cited observations made specifically at the University of Cape Coast (ATL FM, 2020). In addition, all the researchers in the present study are staff of the University of Cape Coast and they had also made cursory observations that the students were not conforming to the COVID-19 preventive measures as imposed by government, and enforced by the university's proctor. The accessible population was the cohort of students from the College of Education Studies who had come to campus to complete their courses (students had to go on break due to the closedown of schools in the middle of the semester at the height of the COVID-19 pandemic), and were living specifically in the university accommodation. The total number of these students was 359 (171 males and 191 females).

\section{Sample Size and Sampling Techniques}

Using Krejcie and Morgan (1970) table for determining sample size for a given population, the ideal sample for the population of 359 is approximately $186[\mathrm{~s}=\mathrm{X} 2 \mathrm{NP}(1-\mathrm{P}) \div \mathrm{d} 2(\mathrm{~N}-1)+\mathrm{X} 2 \mathrm{P}(1-\mathrm{P})]$. In order to get 
Table 1. Age and gender of respondents

\begin{tabular}{|c|c|c|}
\hline Age & $\mathbf{F}$ & $\%$ \\
\hline 18.00 & 31 & 17.0 \\
\hline 19.00 & 39 & 21.3 \\
\hline 20.00 & 39 & 21.3 \\
\hline 21.00 & 29 & 15.8 \\
\hline 22.00 & 19 & 10.4 \\
\hline 23.00 & 4 & 2.2 \\
\hline 24.00 & 5 & 2.7 \\
\hline 25.00 & 5 & 2.7 \\
\hline 26.00 & 4 & 2.2 \\
\hline 27.00 & 2 & 1.1 \\
\hline 29.00 & 2 & 1.1 \\
\hline 30.00 & 2 & 1.1 \\
\hline 32.00 & 1 & 0.5 \\
\hline 41.00 & 1 & 0.5 \\
\hline \multicolumn{3}{|l|}{ Sex } \\
\hline Male & 88 & 48.1 \\
\hline Female & 95 & 51.9 \\
\hline Total & 183 & 100 \\
\hline
\end{tabular}

a proportionate number of male and female respondents, a proportional sampling method was used to target 89 males and 99 females. A convenience sampling method was used to get the final respondents to the questionnaire.

\section{Data Analysis}

A total of 190 questionnaires were distributed for this study and 183 were retrieved which indicates a response rate of $96 \%$. The results are presented in the results and discussion section. The preliminary analysis (A) covers the demographic data of the respondents while Section B looked at the research questions which are presented in frequencies (f) and percentages (\%).

\section{RESULTS AND DISCUSSION}

\section{Preliminary Analyses}

AI. Analysis of the background data of respondents showed that 95 (51.9\%) were females and the rest were males. Most of the respondents were between 19 and 22 years old, and this indicates to a large extent, a true representation of regular undergraduate university students in Ghana. Most of the students enter university by age 18 and for those who go on four-year programmes, they leave at about age 22 . The few $(\sim 10 \%)$ medical students who stay for seven years exit the university around ages 25 . The specific age information is reported in Table 1.

AII. To what extent do students observe the COVID-19 preventive measures?

Participants were asked to specify the extent to which they observe each of the COVID-19 preventive measures under study (handwashing, wearing of nose masks, social distancing, and hand sanitising). These were on a scale of 1 to 4 . With 1 representing Not at All, 2 representing
Sometimes, 3 representing Often and 4 representing Very Often. The results are presented in Table 2 .

The results in Table 2 indicate that respondents differed on the degree or extent to which they practice the COVID-19 preventive measures. Regarding handwashing for example, a considerable number of respondents (39) reported not observing this at all and some others do it in rare cases (89). Only a few respondents agreed that they do wash their hands regularly as needed, in response to the fight against the pandemic. Similar to the current findings, Fielmua, Guba and Mwingyine (2021) studied the hand hygiene and safety behaviours in response to COVID-19 at shopping centres in the Wa township in Ghana, and they reported that adherence to COVID-19 safety protocols at shopping centres were very poor even though the shops had provided the necessary handwashing facilities. Specifically, the researches mentioned that approximately $91.3 \%$ of shoppers did not practice handwashing before entering the shops. On the contrary, Dwipayanti, Lubis, and Harjana (2021) examined the current hand hygiene behaviours during the COVID-19 pandemic, post pandemic behaviour intentions, and the relationship between behaviour, psychosocial and contextual factors among 896 Indonesian citizens over 18 years old. The results were that the majority of respondents did increase their frequency of hand hygiene practices during COVID-19 pandemic. In a related study, Mieth et al. (2021) reported that in their study to examine the prevalence estimate for the compliance with the COVID-19 measures, $94.5 \%$ of participants in their direct questioning group claimed to practice proper hand hygiene, whereas in their indirect questioning group a significantly lower estimate of only $78.1 \%$ was observed. However, the researchers concluded that estimates of the degree of commitment to measures designed to counter the spread of the disease may be significantly inflated by social desirability in direct self-reports. In summary, the present findings coupled with some of the mentioned existing studies suggest that handwashing is an infrequent practice among individuals in the age of COVID-19. It has been shown that, even among nurses handwashing has not always been taken as seriously as it should, with compliance and adherence in clinical settings far from optimal over time (Bezerra et al., 2020; Pittet, 2001). Multiple reports from diverse countries show that hand hygiene compliance rate has been estimated at only $40 \%$ (Erasmus et al., 2010) while the rate of adherence in critical care units is only $46.25 \%$ (Bezerra et al., 2020). Even though handwashing is an easy and lifesaving task, it is not, regrettably, always practiced (Doronina et al., 2017). The present pandemic has drawn attention to handwashing beyond the clinical settings and this focus has to endure.

Most of the respondents in the present study conceded to wearing their nose mask often and very often, although a few (1.1\%) and $12 \%$ reported to not wearing their facemask at all and sometimes respectively. The contextual transfer of face mask use from healthcare settings to public spaces is precisely the aspect of making the "outside world" closely resemble scientific apparatus. Wearing nose masks as a recommended personal protective equipment and as a public health measure to prevent the spread of COVID-19 pandemic, has been deeply

Table 2. Extent to which students observe the basic-four COVID-19 protocols

\begin{tabular}{|c|c|c|c|c|c|c|c|c|}
\hline \multirow{2}{*}{$\begin{array}{l}\text { Extent to which students observe the } \\
\text { following protocols }\end{array}$} & \multicolumn{2}{|c|}{ Not At All } & \multicolumn{2}{|c|}{ Sometimes } & \multicolumn{2}{|c|}{ Often } & \multicolumn{2}{|c|}{ Very Often } \\
\hline & $\mathbf{F}$ & $\%$ & $\mathbf{F}$ & $\%$ & $\mathbf{F}$ & $\%$ & $\mathbf{F}$ & $\%$ \\
\hline Handwashing & 39 & 21.3 & 89 & 46.8 & 48 & 26.2 & 45 & 24.6 \\
\hline Wearing of nose mask & 2 & 1.1 & 22 & 12.0 & 59 & 32.2 & 100 & 54.6 \\
\hline Social distancing & 39 & 21.3 & 89 & 46.8 & 38 & 20.8 & 17 & 9.3 \\
\hline Hand Sanitising & 9 & 4.9 & 59 & 32.2 & 62 & 33.9 & 53 & 29.0 \\
\hline
\end{tabular}


Table 3. Barriers to maintaining social distance

\begin{tabular}{|c|c|c|c|c|}
\hline \multirow{2}{*}{ Statements } & \multicolumn{2}{|c|}{ Disagree } & \multicolumn{2}{|c|}{ Agree } \\
\hline & $\mathbf{F}$ & $\%$ & $\mathbf{F}$ & $\%$ \\
\hline There are more than two occupants in a room and the space is not enough for the recommended social distance & 35 & 19.1 & 148 & 80.9 \\
\hline Keeping a distance from my friends appear hostile & 57 & 31.1 & 126 & 68.9 \\
\hline The nature of course works such as group assignments make it difficult to keep distance from friends & 35 & 19.1 & 183 & 80.9 \\
\hline None of the people I know has been infected so there is no point keeping distance from them & 93 & 50.8 & 90 & 49.2 \\
\hline
\end{tabular}

associated with social and cultural practices and has generated diverse social meanings (see, Martinelli et al., 2021). In spite of the such social and cultural implications, the current findings suggest that most individuals wear their nose mask on a frequent basis. In a related study, Machida et al. (2020) examined the prevalence of wearing masks to prevent COVID-19 and compliance with appropriate measures for the correct use of face masks among the general public in Japan where wearing medical masks is a "cultural" normality. Participants were asked to indicate how often they wore masks for prevention and to what extent they practiced appropriate measures suggested by the World Health Organization. The prevalence of wearing masks was $80.9 \%$ and compliance rates with appropriate measures ranged from $38.3 \%$ to $83.5 \%$. In an alternative account, Purushothaman, Priyangha, and Vaidhyswaran (2020) reported that prolonged use of facemasks induces difficulty in breathing on exertion and excessive sweating around the mouth to the healthcare workers and thus, there is poorer adherence and increased risk of susceptibility to infection. Again, in Fei et al. (2021) the researchers observed that approximately $78 \%$ of the shop attendants they observed did not wear nose masks themselves. The present findings contradict some of the existing reported compliance to the use of nose mask in the fight against COVID-19 spread. In Fei et al.,'s (2020) study among shoppers, it is possible that these shoppers refused to wear their nose mask because they do not interact with other shoppers or do not stay in the shops for long as the case may be in a typical school situation. Agyemang, Agyei-Mensah, and KyereGyeabour (2021) have however reported that most drivers they observed in Ghana, showed a high vulnerability perception to Covid19 , and that older drivers, in particular, consistently wore face masks and insisted on other persons in their commercial vehicles to follow suit. The trend of the results seems to suggest that wearing of nose mask is predominantly practiced by individuals who stay with others at a single location over an extended period of time, such as travellers on a bus, students in school but may not be strictly observed by shoppers who are passing through a location or stay in a certain location with others for a short period of time.

Handsanitising was reported as a somewhat frequent observed protocol among respondents with a greater number of participants agreeing to sanitising their hands often and very often.

However, in a study by Bonful et al. (2020) among transport organisations in Ghana it was reported that almost all stations (93\%) did not have alcohol-based hand sanitizers available for public use (see also, Islam et al., 2021) and that consequently reduces the frequency of hand sanitising practices among people. Herein, students report sanitising their hands frequently so it is possible that even if the university management do not provide hand sanitisers, they probably will purchase their own. Finally, the degree of maintaining social distance was assessed among respondents. Social distancing was the least observed protocol with a significant number of respondents not doing it at all, and others occasionally maintaining a distance. Only a few reported keeping social distance often and very often. In study among residents in North London on their social distancing behaviours, Hills and Eraso (2021) reported that the vast majority (92.8\%) of participants did not adhere to all social distancing rules, and nearly half (48.6\%) engaged in intentional non-adherence of rules.

In summary, the current findings provide a fair knowledge of the extent to which each of the 4-basic government imposed COVID-19 protocols are practiced among university students. The rest of the data reported in the subsequent research question hinges on the factors that impair individual's ability in observing the COVID-19 preventive measures.

AIII: What are the barriers to observing social distancing in efforts to restricting the spread of COVID-19?

In relation to factors that make it difficult to observe the social distancing protocol, the main issues raised were that (1) there are more than two occupants in a room (relatively small room) and the space is not enough for the recommended social distance (2) keeping a distance from friends appear hostile and (3) the nature of coursework (group assignments, groups presentations, and other group works) make it difficult to keep distance from friends. It is worth noting that social distancing is one of the most difficult COVID-19 preventive measures for a collectivistic country like Ghana. In most Ghanaian homes, more than three people share a single room and that in itself does not pave way for social distancing. For example, Ofori (2020) has shown that adequate supply of housing remains a challenge in developing countries, especially Ghana. A room occupancy rate of 5.51 indicates that households are congested and a population of 4,603 accommodated in 496 housing units is evident. That is, even if students want to maintain social distancing, the system in itself makes it undoable. In a related study however, Williams et al. (2020) found that observing social distancing protocols led to a loss of social interaction among residents of the UK, because the suddenness and extensiveness of the lack of face-to-face contact had 'taken a toll' on people, leaving them to feel 'alienated'. The current study also found that despite the factors such as overcrowding in halls, the desire to be perceived as a friendly companion makes it difficult to observe the recommended social distancing protocols even in the absence of factors that students may have no control over such as having more than two roommates. Furthermore, Ghana is classified as a collectivist culture and this is demonstrated by its tight, long-standing dedication to the "group" of the person, whether family or other social ties. This means that most students prefer carrying out their daily activities such as attending lectures, going for lunch, working on assignments, etc, in groups. This 'need to belong' to groups within the school context makes it challenging to adhere to social distancing protocols. Another ritual to maintaining social relationships in Ghana is through the practice of the cultures and norms like warm greetings, handshakes, and hugs. These actions in themselves make it difficult to practice social distancing and the results herein suggest that most people will prioritise their cultural norms over the COVID-19 protocols even if it endangers their lives. For example, the Akan specific aspects of greetings in its basic form, 
Table 4. Hindrances to handwashing practices

\begin{tabular}{|c|c|c|c|c|}
\hline \multirow{2}{*}{ Statement } & \multicolumn{2}{|c|}{ Disagree } & \multicolumn{2}{|c|}{ Agree } \\
\hline & $\mathbf{F}$ & $\%$ & $\mathbf{F}$ & $\%$ \\
\hline \multicolumn{5}{|l|}{ Washing of hands } \\
\hline Inadequate handwashing basins on campus & 70 & 38.3 & 113 & 61.7 \\
\hline There is no need to wash your hands & 162 & 88.5 & 21 & 11.5 \\
\hline One cannot make extra budget to buy additional soap for handwashing & 104 & 56.8 & 79 & 43.2 \\
\hline My friends do not wash their hands frequently too & 79 & 43.2 & 104 & 56.8 \\
\hline Sometimes there is no water or soap in the hand washing stations & 56 & 30.6 & 127 & 69.4 \\
\hline Handwashing basins are located at far distances & 83 & 45.4 & 100 & 54.6 \\
\hline It is difficult to remember washing hands repeatedly & 69 & 37.7 & 114 & 62.3 \\
\hline
\end{tabular}

Table 5. Barriers to hand sanitising practices

\begin{tabular}{|c|c|c|c|c|}
\hline \multirow{2}{*}{ Statements } & \multicolumn{2}{|c|}{ Disagree } & \multicolumn{2}{|c|}{ Agree } \\
\hline & $\mathbf{F}$ & $\%$ & $\mathbf{F}$ & $\%$ \\
\hline The sanitizers have unfriendly smell & 104 & 56.8 & 79 & 43.2 \\
\hline Some of the hand sanitising dispensers do not work well & 62 & 33.9 & 121 & 66.1 \\
\hline There are inadequate hand sanitising dispensers around & 67 & 36.6 & 116 & 63.4 \\
\hline The hand sanitising dispensers are located far & 90 & 49.2 & 93 & 50.8 \\
\hline The sanitisers cause itching around hands & 142 & 77.6 & 41 & 22.4 \\
\hline Dysfunctional hand sanitising dispensers & 88 & 48.1 & 95 & 51.9 \\
\hline Hand sanitisers are costly & 70 & 38.3 & 113 & 61.7 \\
\hline
\end{tabular}

incorporates a verbal component and hand shaking and it is not enough to just stand afar and greet by shouting, or even waving. One can only be pardoned for just waving if he/she has legitimate reasons not to draw nearer for a handshake (see Agyekum, 2004a, 2004b, 2005; Obeng, 1997, 1999; Yankah, 1991, 1995). Greetings in most cases are used as ritualized pre-sequences that come before the actual message in a lot of communicative interactions. They serve as signals, attention getter and preparatory grounds for the possible openings of conversations, discussions and public speeches (see Mey, 1993). Greetings are so frequent and important that when a person enters to greet, normal conversation and other communicative activities are suspended (see Agyekum, 2008) and the aforementioned dominant nature of greetings described here have subtle ways of inhibiting social distancing.

BI. Research Question 1: What are the factors that impair student's abilities to obey the COVID-19 protocol of handwashing?

Research Question 1 sought to find out factors that make it difficult for students to adhere to the government-imposed COVID-19 protocols of hand washing. The results are presented in Table 4.

Table 4 largely suggests that students acknowledge the need to wash their hands in the midst of the pandemic, because most of them disagreed with the notion that there is no need to wash your hands. However, there are key management and a few within-student factors that inhibit this action. First, the respondents admitted to the statement that there are inadequate hand washing stations on campus which presupposes that in some cases a student have to queue to access this facility. They also added that the few handwashing stations are not always resourced, because in many instances there is no water or soap there. The World Bank (2020) have reported that about three billion people around the world have no access to a handwashing facility with water and soap at home and that the small action of frequent handwashing to prevent infection remains out of reach (see also, WaterAid, 2020; WHO/UNICEF, 2019). Thus, vehemently speaking most places in the world including Ghana have no access to water to even help combat the disease spread. Notwithstanding, the respondents also showed that the handwashing stations have been mispositioned. In locating handwashing stations, the amount of space the handwashing station occupies should has to be considered, especially in crowded informal urban settings or camps. The handwashing station has to be strategically placed so they cannot be missed, and they must also be difficult to avoid even during daily routines (e.g., at entrances of buildings, near toilets, etc.). Where possible stakeholders are advised to use locations for handwashing stations that are already accessible for persons with disabilities, i.e., flat level ground, ramps, no steps and door widths that are $800 \mathrm{~mm}$ (UNICEF Fact Sheet, 2020). Unlike certain universities in the Western world and some other places, the university of Cape Coast and many other public universities in Ghana did not have handwashing stations before the COVID-19 pandemic. These facilities came into play following the government's measures to restrict the spread of the virus and the universities seem to be grappling with this new policy of resourcing the campuses with such materials, perhaps due to financial reasons or inadequate expertise in implementing such measures. In addition to these management constraints, there were two critical attitudinal factors that the respondents agreed to: that it is difficult to remember washing hands repeatedly and because their friends do not wash their hands. Looking at the number of courses and assignments required of students, they are more likely not to remember washing their hands since this has not been a practice on campus. Moreover, these students usually are in groups so if the group members do not wash their hands, then there is the implied social influence of the entire group not doing it.

Research Question 2: What are the barriers to handsanitising in efforts to restricting the spread of COVID-19?

From the results presented in Table 5, the main reasons students are unable to sanitise their hands were predominantly management factors of malfunctioning handsanitisers, inadequate handsanitising stations, improper positioning of handsanitising stations. However, one student factor that was mentioned was that the student saw it expensive acquiring their own sanitiser. The COVID-19 has brought about global fall in business and economic hardships (Horowitz, Brown, \& Minkin, 2021) and that will make it difficult for parents to make financial provision to cover hand sanitisers for their wards. Hence, most 
Table 6. Reasons students assign to their inability to wear nose masks

\begin{tabular}{|c|c|c|c|c|}
\hline \multirow{2}{*}{ Statements } & \multicolumn{2}{|c|}{ Disagree } & \multicolumn{2}{|c|}{ Agree } \\
\hline & $\mathbf{F}$ & $\%$ & $\mathbf{F}$ & $\%$ \\
\hline Disposable nose masks are expensive & 115 & 62.8 & 68 & 37.2 \\
\hline Washable nose masks do not last long & 119 & 65 & 64 & 35.0 \\
\hline Wearing masks hides your beauty & 95 & 51.9 & 88 & 48.1 \\
\hline I cannot breathe through the nose masks easily & 66 & 36.1 & 117 & 63.9 \\
\hline Nose masks deform my dressing. & 132 & 72.1 & 51 & 27.9 \\
\hline It is boring to put on nose mask after wearing a nice makeup & 96 & 52.5 & 87 & 47.5 \\
\hline Wearing the nose mask does not help prevent the virus in anyway & 137 & 74.9 & 46 & 25.1 \\
\hline It is irritating to wear the nose masks for extended period of time:(e.g., 2 hours) & 48 & 26.2 & 135 & 73.8 \\
\hline My friends and roommates do not wear their nose masks & 105 & 57.4 & 78 & 42.6 \\
\hline
\end{tabular}

students will depend on the hand sanitisers that are provided by the university.

Research Question 3: Which factors make it difficult for students to wear nose masks in adherence to the COVID-19 preventive measures?

The main reasons that respondents indicated as inhibiting their efforts in wearing the nose masks were the discomfort experienced in the prolonged use of the mask and the fact that they said they could not breath through the masks easily. Quite intriguing, the additional costs evoked by the nose masks was not a barrier to its use. In a previous study of public response to the 2009 influenza A H1N1 pandemic, 71\% of United States respondents supported the recommendation to wear a mask during the flu outbreak. However, in attempts to identify barriers to the wearing of masks among adults in the United States, the most common perceived barrier among the participants was product satisfaction and $85.71 \%$ of the participants agreed that wearing face masks is uncomfortable (Hung, 2018). Rosner (2020) has reported that prolonged use of $\mathrm{N} 95$ and surgical masks by healthcare professionals during COVID-19 has caused adverse effect such as headaches and that has led to poor adherence to nose masks use among the healthcare professionals. In a related study however, Spitzer (2020) says that face masks can prevent the spread of the virus SARS-CoV-2, in particular as this spread can occur from people with no symptoms. However, covering the lower half of the face reduces the ability to communicate, interpret, and mimic the expressions of those with whom we interact. Positive emotions become less recognizable, and negative emotions are amplified. Emotional mimicry, contagion, and emotionality in general are reduced and (thereby) bonding between teachers and learners, group cohesion, and learning - of which emotions are a major driver. Thus, in addition to the health risks imposed by the nose masks.

\section{CONCLUSIONS}

Results from the current study suggest that university students generally observe the four-basic recommended COVID-19 preventive measures, but the extent of practice vary across the four measures. Wearing of nose mask was found to be frequently practiced, whereas hand washing and hand sanitising are largely also observed. However, the degree of adherence to social distancing was quite low and some students do not either do it at all, or in rare circumstances. Regarding the barriers to practicing the preventive measures, the main hinderances to social distance were the management action of taking more than the numbers of students required to make room for social distancing in the halls. Also, the nature of most courses requires such group works as projects, assignments and group presentations and such exercises require that students gather and work together and closely to complete such tasks. Quite intriguing, there was a barrier that bordered on the social implications of maintaining distance from friends. With regards to hand washing, the main barriers were the fact that there are inadequate hand washing stations and the available few have not been situated properly and, in most cases, not resourced. Also, because handwashing practice is a new behaviour they have adopted, there is difficulty in remembering the need to wash hands repeatedly. Like the handwashing stations, it was also found that there are insufficient numbers of hand sanitising stations and again, the locations of such stations are inappropriate.

\section{IMPLICATIONS FOR PRACTICE}

Based on the findings elicited here, it is recommended that the university management trains personnel to manage the handwashing and hand sanitising stations on the campuses, increase the number of hand sanitising and hand washing stations and engage expert advice on the appropriate places to locate these amenities that will be easily accessible and compel students to maintain the protocols. Currently, most course instructions (lectures) span for a period of two hours and some instances, three hours. Since students get uncomfortable with prolonged use of the nose masks, it is suggested that the lecture periods are further broken down such that students are given about ten minutes break after every fifty minuets. Management should make extra efforts to boost finances and ensure that hand sanitising dispensers are well resourced at every time. The digital screen and billboards on the campuses should project teasers on the protocols to COVID-19 prevention in order to remind and sensitise students on the need to adhere to them. Finally, the current room intake in the university halls could be reduced to a number that will make it possible for students to maintain social distance.

Author contributions: All authors were involved in concept, design, collection of data, interpretation, writing, and critically revising the article. All authors approve final version of the article.

Funding: The authors received no financial support for the research and/or authorship of this article.

Declaration of interest: Authors declare no competing interest.

Data availability: Data generated or analysed during this study are available from the authors on request.

\section{REFERENCES}

Agyekum, K. (2004). Aspects of Akan persuasive language. RASKInternational Journal of Language and Communication, 21, 63-96. 
Agyekum, K. (2004). The socio-cultural concept of face in Akan communication. Pragmatics \& Cognition, 12(1), 71-92. https://doi.org/10.1075/pc.12.1.06agy

Agyekum, K. (2005). An Akan oral artist-the use of proverbs in the lyrics of Kwabena Konadu. Institute of African Studies Research Review, 21(1), 1-17. https://doi.org/10.4314/rrias.v21i1.22929

Agyekum, K. (2008). The pragmatics of Akan greetings. Discourse Studies, 10(4), 493-516. https://doi.org/10.1177/146144560809 1884

Agyemang, E., Agyei-Mensah, S., \& Kyere-Gyeabour, E. (2021). Face mask use among commercial drivers during the COVID-19 pandemic in Accra, Ghana. Journal of Community Health, 1-10. https://doi.org/10.1007/s10900-021-01004-0

Bezerra, T. B., Valim, M. D., Bortolini, J., Ribeiro, R. P., Marcon, S. R., \& Moura, M. E. B. (2020). Adherence to hand hygiene in critical sectors: Can we go on like this? Journal of Clinical Nursing, 29(1314), 2691-2698. https://doi.org/10.1111/jocn.15293

Bonful, H. A., Addo-Lartey, A., Aheto, J. M. K., Ganle, J. K., Sarfo, B., \& Aryeetey, R. (2020). Limiting spread of COVID-19 in Ghana: Compliance audit of selected transportation stations in the greater accra region of Ghana. PloS One, 15(9), e0238971-e0238971. https://doi.org/10.1371/journal.pone.0238971

Chen, N., Zhou, M., Dong, X., Qu, J., Gong, F., Han, Y., Qiu, Y., Wang, J., Liu, Y., Wei, Y., Xia, J., Yu, T., Zhang, X., \& Zhang, L. (2020). Epidemiological and clinical characteristics of 99 cases of 2019 novel coronavirus pneumonia in Wuhan, China: a descriptive study. Lancet, 395(10223), 507-513. https://doi.org/10.1016/S01406736(20)30211-7 PMID:32007143 PMCID:PMC7135076

Coronaviridae Study Group of the International Committee on Taxonomy of Viruses. (2020). The species severe acute respiratory syndrome-related coronavirus: Classifying 2019-nCoV and naming it SARS-CoV-2. Nature Microbiology, 5(4), 536-544. https://doi.org/10.1038/s41564-020-0695-z

Doronina, O., Jones, D., Martello, M., Biron, A., \& Lavoie-Tremblay, M. (2017). A systematic review on the effectiveness of interventions to improve hand hygiene compliance of nurses in the hospital setting. Journal of Nursing Scholarship, 49(2), 143-152. https://doi.org/10.1111/jnu.12274

Dwipayanti, N. M. U., Lubis, D. S., \& Harjana, N. P. A. (2021). Public perception and hand hygiene behavior during COVID-19 pandemic in indonesia. Frontiers in Public Health, 9, 621800-621800. https://doi.org/10.3389/fpubh.2021.621800

Erasmus, V., Daha, T. J., Brug, J., Richardus, J. H., Behrendt, M. D., Vos, M., \& van Beeck, E. F. (2010). Systematic review of studies on compliance with hand hygiene guidelines in hospital care. Infection Control and Hospital Epidemiology, 31(3), 283-294. https://doi.org/10.1086/650451

Fielmua, N., Guba, B. Y., \& Mwingyine, D. T. (2021). Hand hygiene and safety behaviours at shopping centres in COVID-19: An observation in wa township in Ghana. Journal of Water, Sanitation, and Hygiene for Development, 11(3), 442-452. https://doi.org/10.2166/washdev.2021.240

Ghana Health Services. (2020). Situation update, Covid-19 outbreak in Ghana as at 2 July 2020. https://ghanahealthservice.org/covid19/ archive.php
Gorbalenya, E. A., Baker, S. C., Ralph, S. B., Groot, R. J., Drosten, C., A., Gulyaeva, B. L., Haagmans, B. L., Lauber, C., Leontovich, A. M., Neuman, B. W., Penzar, D., Perlman, S., Poon, L. L. M., Samborskiy, D., Sidorov, I. A., Sola, I., \& Ziebuhr, J. (2020). Severe acute respiratory syndrome-related coronavirus: The species and its viruses - a statement of the Coronavirus Study Group. Nature Microbiology, 5, 536-544. https://doi.org/10.1038/s41564-0200695-z

Hills, S., \& Eraso, Y. (2021). Factors associated with non-adherence to social distancing rules during the COVID-19 pandemic: A logistic regression analysis. BMC Public Health, 21(1), 1-25. https://doi.org/10.1186/s12889-021-10379-7

Holmes, E. (2020). Novel-2019 coronavirus genome. http://virological.org/t/novel-2019-coronavirus-genome/319

Hung, Y. (2018). A study of barriers to the wearing of face masks by adults in the US to prevent the spread of influenza. Arizona State University.

Leung, N. H. L., Chu, D. K. W., Shiu, E. Y. C., Chan, K., McDevitt, J. J., Hau, B. J. P., Yen, H., Li, Y., Ip, D. K. M., Peiris, J. S. M., Seto, W., Leung, G. M., Milton, D. K., \& Cowling, B. J. (2020). Respiratory virus shedding in exhaled breath and efficacy of face masks. Nature Medicine, 26(5), 676-680. https://doi.org/10.1038/s41591-020-0843-2

Li, C., Li, H., Li, Q., Li, D., Guan, X., Wu, J. T., Wu, Y., Wu, P., Wang, R., Wang, Q., Wang, X., Zhou, L., Zhou, S., Tong, Y., Ren, R., Leung, G. M., Leung, K. S. M., Lau, E. H. Y., Wong, J. Y., . . Feng, Z. (2020). Early transmission dynamics in wuhan, china, of novel Coronavirus-Infected pneumonia. The New England Journal of Medicine, 382(13), 1199-1207. https://doi.org/10.1056/ NEJMoa2001316

Machida, M., Nakamura, I., Saito, R., Nakaya, T., Hanibuchi, T., Takamiya, T., Odagiri, Y., Fukushima, N., Kikuchi, H., Kojima, T., Watanabe, H., \& Inoue, S. (2020). Adoption of personal protective measures by ordinary citizens during the COVID-19 outbreak in Japan. International Journal of Infectious Diseases, 94, 139-144. https://doi.org/10.1016/j.ijid.2020.04.014

Martinelli Lucia, Kopilaš Vanja, Vidmar Matjaž, Heavin Ciara, Machado Helena, Todorović Zoran, Buzas Norbert, Pot Mirjam, Prainsack Barbara, Gajović Srećko (2021). Face masks during the COVID-19 pandemic: A simple protection tool with many meanings. Frontiers in Public Health, 8, 947. https://doi.org/10.3389/fpubh.2020.606635

Martinelli, L., Kopilaš, V., Vidmar, M., Heavin, C., Machado, H., Todorović, Z., Buzas, N., Pot, M., Prainsack, B., \& Gajović, S. (2020, 2021). Face masks during the COVID-19 pandemic: A simple protection tool with many meanings. Frontiers in Public Health, 8 , 606635-606635. https://doi.org/10.3389/fpubh.2020.606635

Mieth, L., Mayer, M. M., Hoffmann, A., Buchner, A., \& Bell, R. (2021). Do they really wash their hands? Prevalence estimates for personal hygiene behaviour during the COVID-19 pandemic based on indirect questions. BMC Public Health, 21(1), 1-8. https://doi.org/10.1186/s12889-020-10109-5

Ofori, P. (2020). Housing poverty in developing countries: challenges and implications for decent accommodation in Swedru, Ghana. Acta Structilia, 27(2), 57-92. https://doi.org/10.18820/24150487/ as $27 \mathrm{i} 2.3$ 
Pittet, D. (2001). Improving adherence to hand hygiene practice: a multidisciplinary approach. Emerging Infectious Diseases, 7(2), 234. https://doi.org/10.3201/eid0702.010217

Purushothaman, P. K., Priyangha, E., \& Vaidhyswaran, R. (2021). Effects of prolonged use of facemask on healthcare workers in tertiary care hospital during COVID-19 pandemic. Indian Journal of Otolaryngology and Head \& Neck Surgery, 73(1), 59-65. https://doi.org/10.1007/s12070-020-02124-0

Rosner, E. (2020). Adverse effects of prolonged mask use among healthcare professionals during COVID-19. Journal of Infectious Diseases of Epidemiology, 6(3), 130. https://doi.org/10.23937/24743658/1510130

Spitzer, M. (2020). Masked education? The benefits and burdens of wearing face masks in schools during the current Corona pandemic. Trends in Neuroscience and Education, 20, 100138. https://doi.org/10.1016/j.tine.2020.100138
UNICEF. (2020). Handwashing with soap, critical in the fight against coronavirus, is 'out of reach' for billions.

Williams, S. N., Armitage, C. J., Tampe, T., \& Dienes, K. (2020). Public perceptions and experiences of social distancing and social isolation during the COVID-19 pandemic: A UK-based focus group study. BMJ Open, 10(7), e039334. https://doi.org/10.1136/bmjopen-2020039334

World Health Organization (WHO). (2020a). Coronavirus disease 2019 (COVID-19) Situation Report -10. https://www.worldbank.org/en/ news/feature/2020/04/30

World Health Organization (WHO). (2020b). Rational use of personal Protective Equipment (PPE) for coronavirus disease (COVID-19): Interim guidance World Health Organization (No. WHO/2019-nCoV/IPC PPE_use/2020.2). 